\title{
Serum Magnesium in Diabetes Mellitus
}

\author{
Rahul S. Patil, R. J. Kapale, Dany P. Jhon, Gosavi Rohit, Pramod Kulkarni \\ Department of Medicine, Krishna Institute of Medical Sciences Deemed To Be University, Karad, India.
}

Section: Healthcare

Sci. Journal Impact

Factor: $6.1(2018)$

ICV: 90.90 (2018)

(c) (i) (8)

Copyright@IJCRR

\section{ABSTRACT}

Introduction: Diabetes mellitus is the most common endocrine disorder, encountered in practice. The incidence of diabetes is increasing at a rapid pace and is evolving into an epidemic.

Methodology: In the present study, serum magnesium levels of 100 diabetic patients are compared with 30 nondiabetic patients with age and sex-matched controls. Serum magnesium levels are significantly low amongst diabetics as against nondiabetic controls giving the incidence of hypomagnesemia in diabetics to be $66 \%$.

Results: Amongst diabetics serum magnesium levels are adversely influenced by factors like male gender, longer duration of diabetes, poor glycemic control, higher serum cholesterol levels, hypertension as well as micro and macrovascular complications showing significant prevalence. Values of serum magnesium are significantly low in patients with microvascular complications as against those with macrovascular complications. Diabetic ketoacidosis is significantly associated with lower serum magnesium levels. However, age group, type of diabetes, presence of infection do not influence serum magnesium levels in the present study group.

Conclusion: consider magnesium deficiency as a prognostic factor influencing the progression of diabetic complications and justify the therapeutic role of magnesium supplementation in achieving the reduction in insulin resistance, better glycemic control and slowing down of progressive diabetic complications.

Key Words: Serum, Magnesium, Diabetes Mellitus

\section{INTRODUCTION}

Diabetes mellitus is the most common endocrine disorder, encountered in practice. The incidence of diabetes is increasing at a rapid pace and is evolving into an epidemic. The worldwide prevalence of diabetes mellitus has risen dramatically over the past two decades, from an estimated 30 million cases in 1985 to 177 million cases in 2000. Based on current trends more than 360 million individuals will have diabetes by year $2030 .{ }^{1}$

The restricted diets which most diabetics must follow do not allow them to meet the recommended daily allowances of micronutrients like zinc, copper, chromium, manganese, and magnesium regarding diabetes mellitus remains controversial with many questions unanswered.

Magnesium, one of the abundant intracellular cation has many wider metabolic implications as it relates to diabetes mellitus.
Hypomagnesemia has long been known to be associated with diabetes mellitus. Magnesium is an essential cofactor in both glucose transporting mechanisms of cell membranes and more than 300 enzymes in carbohydrate metabolism. Thus the association of diabetes and hypomagnesaemia is significant for its wide-ranging impact on diabetic control, glycosuria, atherosclerosis, dyslipidemia, metabolic, microvascular and macrovascular complications. Magnesium supplementation is safe and effective for the improvement of diabetic complications. However no supplements can replace insulin for patients with type -1 diabetes, even in type 2 diabetes these supplements may only reduce rather than eliminate medication needs. ${ }^{1}$

We know that diabetes is a progressive disorder and complications are inevitable. People with severe hyperglycemia can remain largely asymptomatic for a long period. Consequently, at the time of diagnosis of diabetes, many

\section{Corresponding Author:}

Dr. Mrs. R. J. Kapale, Department of Medicine, Krishna Institute of Medical Sciences Deemed To Be University, Karad, India. Email: hodmedicine@kimsuniversity.in

ISSN: 2231-2196 (Print)

Received: 18.07.2020
ISSN: $0975-5241$ (Online)

Revised: 26.08 .2020
Accepted: 06.09.2020

Published: 22.09 .2020 
of them have some end-organ damage. Thus the enormous impact of diabetes on morbidity and early mortality in western as well as developing countries underscores the importance of closely examining all possible factors in pathogenesis. ${ }^{2,3}$

Hence only hope to lessen the burden of diabetes is the universal screening of diabetes so that early detection and intervention is possible. So we are studying here serum magnesium levels in diabetes mellitus.

\section{MATERIALS AND METHODS}

Hundred diabetic patients attending diabetics clinic and admitted in medicine wards throughout one and half year are included in this study.

\section{Inclusion criteria}

Diabetic patients randomly selected irrespective of age, sex, type of diabetes, duration, glycemic control, with or without diabetic complications. Thirty age and sex-matched nondiabetic controls.

\section{Exclusion criteria}

Patients with malabsorption, nephrolithiasis, hyperthyroidism, hyperparathyroidism, malignancy, chronic renal failure: i.e., the conditions mainly influencing serum magnesium levels.

Patients who have gone through gastrointestinal tasks and nephrectomy. Patients on hormones other than insulin just as on diuretics, anticonvulsants, chemotherapy and immunosuppressants.

\section{OBSERVATIONS AND RESULTS}

There is a highly significant difference in mean values of serum magnesium levels as observed in diabetic and nondiabetic individuals. This provided us with a normal range of serum magnesium as $1.892-2.008 \mathrm{mg} / \mathrm{dl}$. In the present study, the values below $1.8 \mathrm{mg} / \mathrm{dl}$ are considered as low.

In this, there are 66 diabetic patients and 8 non-diabetic patients (controls) whose serum magnesium level is $\leq 1.892$ and 34 diabetic patients and 22 non-diabetic patients (controls) whose serum magnesium level is $>1.892$.

Comment: Statistically significant low Serum magnesium levels are found in diabetics as compared to age and sexmatched non-diabetic controls. $(\mathrm{P}<0.01)$

\section{Sexwise distribution of serum magnesium in diabetes}

This table is showing 22 diabetic female having low serum magnesium level out of 41 diabetic female and 44 di-

abetic male having low serum magnesium level out of 59 diabetic male. Serum magnesium levels are significantly low in male diabetics as compared to female diabetics. $(\mathrm{P}>0.05)$

\section{Correlation of serum magnesium with the type of diabetes mellitus}

Type of diabetes is not found to have any influence on serum magnesium levels. $(\mathrm{P}>0.05)$.

\section{Correlation of serum magnesium with the dura- tion of diabetes mellitus}

Longer duration of diabetes has a statistically significant influence on low levels of serum magnesium in diabetic subjects under study. $(\mathrm{P}<0.01, \mathrm{P}<0.05, \mathrm{P}>0.05)$

\section{Correlation of serum magnesium with glycemic control in diabetics}

Association of poor glycemic control with low serum magnesium level was found to be statistically highly significant. $(\mathrm{P}<0.01)$

\section{Correlation of serum magnesium with serum cholesterol in diabetics}

Association of low serum magnesium level with high cholesterol levels in diabetes is highly significant. $(\mathrm{P}<0.01)$

\section{Correlation of serum magnesium with compli- cations of diabetes mellitus}

Association of low serum magnesium level with single/multiple complications is statistically significant as compared to those without complications. $(\mathrm{P}<0.01, \mathrm{P}>0.05, \mathrm{P}<0.01)$

\section{Correlation of serum magnesium with diabetic retinopathy}

Serum magnesium levels are significantly low in diabetics with retinopathy as compared to those without retinopathy. $(\mathrm{P}<0.01)$

\section{Correlation of serum magnesium with diabetic nephropathy}

Serum magnesium levels are significantly low in diabetics with nephropathy as compared to those without nephropathy. $(\mathrm{P}<0.01)$

\section{Correlation of serum magnesium with diabetic neuropathy} Serum magnesium levels are significantly low in diabetics with neuropathy as against those without neuropathy. $(\mathrm{P}<0.05)$ 


\section{Correlation of serum magnesium with hyper- tension in diabetes mellitus}

Serum magnesium levels are significantly low amongst diabetics with hypertension as compare to those without hypertension. $(\mathrm{P}<0.05)$

\section{DISCUSSION}

Trace elements are uniquely required for growth and maintenance of life and health. Lack or inadequate supply of such nutrients produces a functional impairment or can result in disease. The clinical significance and evaluation of trace elements such as zinc, copper, chromium, manganese regarding different diseases including diabetes mellitus remain controversial. One of the most abundant of earth's metallic elements and quantitatively the fourth most plentiful cation in vertebrates, magnesium has many wider metabolic implications as it relates to diabetes mellitus.

\section{Association of Hypomagnesemia and Diabe- tes Mellitus.}

The present study includes 30 nondiabetic controls with serum magnesium values ranging from 1.7 to $2.3 \mathrm{mg} / \mathrm{dl}$, with a mean of $1.95 \mathrm{mg} / \mathrm{dl}$. 100 diabetic subjects showed serum magnesium values ranging from 1 to $2.2 \mathrm{mg} / \mathrm{dL}$. with a mean of $1.649 \mathrm{mg} / \mathrm{dL}$, The difference between mean values is highly significant and this provides us with a normal range of serum magnesium as 1.892 to $2.008 \mathrm{mg} / \mathrm{dL}$. Thus the values of serum magnesium below $1.8 \mathrm{mg} / \mathrm{dL}$ are considered as abnormal (low serum magnesium).

Out of 100 diabetic cases, 66 were found to have low serum magnesium, which is statistically significant on comparing with age and sex-matched non-diabetic controls. This resulted in an incidence of hypomagnesemia to be $66 \%$ in our study. There are studies in the literature that have reported hypomagnesemia in diabetes mellitus.

In diabetics, plasma magnesium concentrations have been reported to be lower.

\section{Serum Magnesium and Age Group Of Dia- betic Patients}

There was no influence of age on serum magnesium in diabetic patients in the present study. V.K.Srivastava et al had also reported no significant difference in serum magnesium levels in various age "groups.

\section{Serum Magnesium and Sex wise Distribution In Diabetes Mellitus.}

In the present study out of 59 diabetic males, 44 showed low serum magnesium and out of 41 female diabetic patients, 22 showed low serum magnesium and the levels were sig- nificantly low in male diabetics as against female diabetics. However, this has not been substantiated by the literature reviewed. Smith et $\mathrm{al}^{1}$ stated in their study on diabetic patients who were treated with insulin and those not with insulin that men had a significantly higher concentration of magnesium than women in the non-insulin treated group.

Brown, I R. et al. ${ }^{2}$ observed a direct relationship between the excretion of both calcium and magnesium and glycosylated $\mathrm{Hb}$ in female patients $(\mathrm{P}<0.01)$ but not in males who had similar glycosylated $\mathrm{Hb}$ values. He also studied the sex difference in the relationship of calcium and magnesium excretion to glycemic control in type I diabetes and found that in poorly controlled patients, females may have a greater risk than males of developing the complications associated with chronic calcium and magnesium loss.

\section{Serum Magnesium and Type Of Diabetes Mel- litus}

The present study includes total 68 patients with diabetic type 2 and 32 patients with diabetes type 1 disease were included out of which $61.8 \%$ of diabetes type 2 and $75 \%$ of diabetes type 1 subjects show low serum magnesium levels. ${ }^{5}$ However, when subjected to statistical analysis, the type of diabetes was not found to have any influence on serum magnesium levels. (Table No. 4, Graph No.3).

Kao WH et $\mathrm{al}^{3}$ studied serum and dietary magnesium and the risk for type 2 diabetes mellitus. Amongst white participants a graded inverse relationship between serum magnesium levels. Type 2 diabetes has been observed. In contrast, little or no such association has been observed in black participants. Amongst white participants, low serum magnesium level is a strong and independent predictor of future development of type 2 diabetes mellitus.

\section{Serum Magnesium With Duration Of Diabetes}

The present study shows that longer duration of diabetes mellitus has a significant influence on serum magnesium levels. The groups with a duration of diabetes $>5$ years are showing an increased number of cases with low serum magnesium as compared to the group with the duration of diabetes $<5$ years.

Longer duration of diabetes is associated with a higher incidence of complications due to the longer duration of uncontrolled diabetic status associated with lower magnesium levels.

\section{Serum Magnesium and Glycemic Control In Diabetics}

The association of poor glycemic control i.e. (Fasting BSL $>140$ / or Postprandial BSL > $200 \mathrm{mg} / \mathrm{dL}$ ) with low serum magnesium level is found to be statistically highly significant in our study group. Several studies in the literature have 
suggested an association between magnesium depletion and insulin resistance and/ or reduction of insulin secretion in these cases.

Lima et $\mathrm{al}^{4}$ studied 128 subjects with type 2 diabetes after oral magnesium supplementation for metabolic control where magnesium tended to increase plasma, cellular and urine magnesium and caused a significant fall in serum fructosamine level and concluded that magnesium depletion is common in poorly controlled patients of type 2 diabetes mellitus.

\section{Serum Magnesium and Serum Cholesterol Levels In Diabetes}

The present study shows a significant association in low serum magnesium level with high cholesterol levels in diabetics. V.K.Srivastava et $\mathrm{al}^{5}$ showed a similar inverse relationship between serum magnesium and serum cholesterol.

Corica F. et $\mathrm{al}^{6}$ studied effects of oral magnesium supplementation on plasma lipid concentrations in patients with diabetes type 2 and suggested that oral supplementation of magnesium may be useful in the treatment of hyperlipidemia in patients with diabetes type 2 .

\section{Serum Magnesium and Various Complica- tions of Diabetes Mellitus}

The present study includes 100 diabetics with 60 patients with multiple complications (micro / macrovascular,/ metabolic), 17 patients with single complication and 23 diabetics are without any appeared complication.

The association of low serum magnesium level with single or multiple complications is highly significant as against those without complications. The complications include diabetic retinopathy, nephropathy, neuropathy, hypertension, ischemic heart disease, cerebrovascular accident, peripheral vascular disease, infections, diabetic ketoacidosis and hypoglycemia.

In our study, there is a significant association of hypomagnesemia with diabetic retinopathy, nephropathy, neuropathy, hypertension coronary artery disease and diabetic ketoacidosis. A number of metabolic abnormalities and pathological changes that are associated with diabetes include ${ }^{7}$

Grafton et $\mathrm{al}^{8}$ has focussed on the inositol transport theory. The theory suggests that hyperglycemia induces the increased activity of the enzyme aldose reductase, which leads to the intracellular accumulation of sorbitol. The accumulated sorbitol inhibits inositol transport, leading to a decrease in intracellular inositol and inhibition of sodiumpotassium ATPase activity. The data of Grafton et al shows that hypomagnesemia causes a decrease in the affinity of the inositol transport protein for inositol, leading to a twofold reduction in the rate of inositol transport. This work links hy- pomagnesemia and inositol theory which links diabetes and its vascular complications.

\section{Serum Magnesium and Diabetic Retinopathy}

In our study, out of 44 patients with diabetic retinopathy, 40 are associated with low serum magnesium. Hypomagnesemia has been demonstrated in patients with diabetic retinopathy, with lower magnesium levels predicting a greater risk of severe diabetic retinopathy, the insulin-treated diabetic patients who had the disease for 10 years and grouped the patients into 2 subgroups according to the severity of retinopathy and reported definite hypomagnesemia that was most pronounced in the subgroup having the severest degree of retinopathy. Thus the study states that hypomagnesemia appears to be an additional risk factor in the development and progression of this complication. ${ }^{9}$

\section{Serum Magnesium and Diabetic Nephropa- thy}

Our study includes 35 patients with recently detected diabetic nephropathy (having albuminuria, $24 \mathrm{hr}$ urinary protein $>150 \mathrm{mg} \%$ and normal renal function tests) and 31 of them show low serum magnesium which is statistically significant. ${ }^{10}$

Allegra A et $\mathrm{al}^{10}$ evaluated plasma, erythrocyte and platelet magnesium levels in patients with diabetes type 1 with normoalbuminuria, microalbuminuria and clinical proteinuria in a group of healthy subjects and found-

\section{Serum Magnesium and Diabetic Neuropathy}

In our study, out of 31 patients with evidence of diabetic neuropathy, 25 are showing low serum magnesium level and the association is statistically significant.

The study by Lima et $\mathrm{al}^{4}$ on 128 patients Type 2 Diabetes mellitus concluded that magnesium depletion was common in poorly controlled patients with type 2 diabetes especially in those with neuropathy or coronary disease. The fact was also supported by T Cruz et al. ${ }^{11}$

\section{Serum Magnesium and Hypertension and Ischemic Heart Disease and Diabetes}

Out of 21 patients of diabetes with hypertension 18 have low serum magnesium and out of 14 patients of diabetes with IHD 13 have low serum magnesium which is statistically significant. Magnesium concentration found in diabetes type 2 and hypertensive patients may result in a defective tyrosine-kinase activity at the insulin receptor level and exaggerated intracellular Calcium concentration, both events responsible for impairment in insulin action and worsening of insulin resistance in NIDDM and hypertensive patients.

Resnik $^{12}$.proposed that thes primary defect present in all organ systems is an abnormality of cellular ion handling. 


\section{Serum Magnesium and Infections in Diabe- tes Mellitus}

Our study includes 13 patients of diabetes with infections but the statistical analysis does not reveal significant influence on serum magnesium levels.

\section{Serum Magnesium and Diabetic Ketoacido- sis}

The study has 13 patients of diabetes presenting with ketoacidosis out of which 12 have hypomagnesemia at the time of ketosis, the results being significant, on statistical analysis.

Morris $\mathrm{R}$ et $\mathrm{al}{ }^{13}$ has done the study in diabetic cats with ketoacidosis and found the urinary fractional excretion of magnesium significantly higher in cats with diabetes when compared with controls.

\section{CONCLUSIONS}

Serum magnesium levels are significantly low in diabetics as against age and sex-matched non-diabetic individuals (control). Age has no significant influence on serum magnesium levels in diabetes. Serum magnesium levels are significantly low in male diabetics compared to female diabetics in the present study. Longer duration of diabetes mellitus is found to be significantly associated with decreasing levels of serum magnesium, in this study. Poor glycemic control is significantly associated with low serum magnesium levels. Higher serum cholesterol levels are found to have a significant association with low serum magnesium levels in diabetics. In the present study, retinopathy and nephropathy are commonest complications, neuropathy being next in order while hypertension, ischemic heart disease are common compared to cerebrovascular accident and peripheral vascular disease which are found to be uncommon. The association of low serum magnesium levels with multiple complications of diabetes is found to be statistically significant. Serum magnesium levels are significantly low in diabetics with retinopathy compared to those without retinopathy. Serum magnesium levels are significantly low in diabetics with nephropathy (incipient) compared to those without nephropathy. The association of low serum magnesium levels with diabetic neuropathy is found to be statistically significant. The serum magnesium levels are significantly low among diabetics with hypertension as against those without hypertension. The association of low serum magnesium levels in diabetics with ischemic heart diseases is found to be statistically significant. Infections do not influence the serum magnesium level's in diabetics. Amongst patients with diabetic ketoacidosis, serum magnesium levels are significantly low as against those without ketoacidosis.

Acknowledgement: Authors acknowledge the immense help received from the scholars whose articles are cited and included in references to this manuscript. The authors are also grateful to authors/editors/publishers of all those articles, journals and books from where the literature for this article has been reviewed and discussed.

Conflict of interest: Nothing to report.

Source of funding: Kimsdu Karad

\section{REFERENCES}

1. Yajnik CS, Smith RF, Hockaday TD, Ward Nl, Fasting plasma magnesium concentrations and glucose disposal in diabetes. BMJ 1984:288: 1032-1034.

2. Brown IR, McBain AM, Chalmers J. Campbell IW, the Sex difference in the relationship of calcium and magnesium excretion to glycemic control in type I diabetes mellitus. Clinica chima acta 283 (1-2) : 1999 May 119-28

3. Kao W H, Folsom AR, Nieto FJ Watson RL, Brancati FL, Serum and dietary magnesium and the risk for type 2 Diabetes Mellitus: the atherosclerosis risk in communities study. Archives of Int Medicine 1999 Oct, 159 (18): 2151-9.

4. V.K.Srivastava, A.K.Chauhan, V.LLahiri, The significance of serum magnesium in diabetes mellitus. Indian Journal of Medical Sciences, May 1993 47(5): Page 119-122.

5. Corica F. Allegra A. Buemi M. lentile R.Consonello A. Bonanzinga S. Macaione S.Ceruso D. : Obesity and Hypertension the role of magnesium Recent. Progress in Medicina 1998 Apr 89 (4): 169-76.

6. Grafton G, Baxter MA, The role of magnesium in diabetes mellitus.J Diabetes Complications 1992:6:143-149.

7. McNair P, Christiansen C. Madsbad S. Hypomagnesemia, a risk factor in diabetic retinopathy. Diabetes 1978 : 27: 1075 - 1077

8. Allegra A. CorsonelloA. Buemi M., D. Angelo R. di Bere ctetto A. Bonaszinga S.Cluinolto D. lestile R. Corica F. Journal of Trace Elements in Medicine and Biology 1997 Nov. 11(3): 154 -7 .

9. T. Cruz et al. : The effect of magnesium supplementation in increasing doses on the control of type 2 diabetes. Diabetes Care 21: 682-686.

10. Resnick LM.: Cellular calcium and magnesium metabolism in the pathophysiology and treatment of hypertension and related metabolic disorders. Am J Med 1992-93 (Suppl 2A): ZA 1152A 204.

11. Norris CR., Nelson RW.,Christopher MM. Serum total and ionized magnesium concentration and urinary fractional excretionof magnesium in cats with diabetes and diabetic ketoacidosis. Journal of American Veterinary Medical Association 1999 Nov. 15,215 (10): 1455-9. 